\title{
Promises Made to Be Broken: Performance and Performativity in Digital Vaccine and Immunity Certification
}

\author{
Stefania MILAN*, Michael VEALE***iD, Linnet TAYLOR***(D) and \\ Seda GÜRSES****
}

\begin{abstract}
Digital vaccination certification involves making many promises, few of which can realistically be kept. In this paper, we demonstrate how this phenomenon constitutes various forms of theatre - immunity theatre, border theatre, behavioural theatre and equality theatre - doing so by drawing on perspectives from technology regulation, migration studies and critical geopolitics. Technological theatre and political theatre often serve valid functions, but these forms are problematic for several reasons. First, they involve real-world infrastructures that, while unlikely to accomplish the task at hand, will nevertheless last a long time and be repurposed. They therefore constitute governance by data infrastructure, diverting action and control away from elected legislators to for-profit contractors. Second, vaccine certification effectively legitimises inequalities between countries and people by formalising ways to distinguish between the vaccinated and non-vaccinated and to exclude the latter, thus reinforcing both mobility and connectivity divides. It serves as a way to (further) close borders and to regulate, through code and infrastructure, access to public goods such as employment and public space. Finally, the project of certification displaces a more important action, namely addressing the radical inequality in countries' ability to combat the pandemic.
\end{abstract}

\section{INTRODUCTION}

On 17 March 2021, the European Commission unveiled its much-awaited proposal for the "vaccination pass", termed the Digital Green Certificate. The certificate would provide "proof" of health status with respect to COVID-19 to "facilitate safe free movement" across the European Union (EU). In "digital or paper format", the pass is expected to include machine-readable elements such as QR codes and digital signatures "to allow

\footnotetext{
* University of Amsterdam, The Netherlands.

** University College London, UK.

*** TILT, Tilburg University, The Netherlands; email: 1.e.m.taylor@tilburguniversity.edu. Funding acknowledgments: Linnet Taylor - Horizon 2020 ERC StG 757247; Stefania Milan - Horizon 2020 ERC StG 639379.

***** TU Delft, The Netherlands.
} 
the sharing of electronic signature keys so that the authenticity of Digital Green Certificates can be verified across the EU". ${ }^{1}$ This move mirrors other initiatives around the world to certify, at the very least, receipt of an authorised COVID-19 vaccine. These include Israel, whose "Green Pass" was deployed in early 20212; Hungary and Iceland ${ }^{3}$; and proposals in the UK ${ }^{4}$ and the USA. ${ }^{5}$ In Israel, Hungary and Iceland, certification is also available to those who have tested positive for the virus, but it is unclear whether other countries planning certification systems will also go in this direction. The main push for certification has been economically motivated, in the EU's case with Southern European countries pushing for certification in order to maximise the chance that they can receive international tourists during the 2021 summer holiday season, ${ }^{6}$ and in the case of the UK with the Conservative government pushing for it as a way for sectors such as hospitality and sport to reopen. ${ }^{7}$ In this article, we primarily use the term "immunity certification" as a broader term covering programmes worldwide, some of which aim to certify that people are vaccinated, but many of which focus on the notion of immunity, including having tested positive in the past for COVID-19.

On the global level, the ID2020 Alliance, a for-profit initiative that counts Microsoft and Accenture among its members and purports to "set the future course of digital identity", has launched its Good Health Pass, with the ambition of creating "a blueprint for interoperable digital health pass systems". ${ }^{8}$ Notably, this proposal has been criticised even by members of ID2020's own board ${ }^{9}$ on the basis that it is a techno-solutionist move that furthers the business interests of the alliance's partners while ignoring scientific evidence and the risks to human rights. Critique also came from the World Health Organization (WHO), which issued a warning early in the pandemic that certification of immunity was not supported by the scientific evidence. ${ }^{10}$ With the impending launch of the EU's Digital Green Certificate, the

\footnotetext{
1 European Commission, "Further crucial step for the Digital Green Certificate" (European Commission, 2021) $<$ https://ec.europa.eu/commission/presscorner/detail/en/IP_21_1883> (last accessed 4 May 2021).

2 Government of Israel, "Green Pass, Vaccination Certificate and Certificate of Recovery" (GOV.IL, 2021) <https:// www.gov.il/en/Departments/General/corona-certificates> (last accessed 22 April 2021).

3 CNN, “Immunity passports' are already here. But they come with warnings" (2021) < https://edition.cnn.com/ travel/article/hungary-iceland-covid-immunity-passport-scn/index.html> (last accessed 22 April 2021).

4 Reuters, "UK mulling vaccine passport options for at home and abroad, minister says" (Reuters.com, 17 March 2021) < https://www.reuters.com/article/uk-health-coronavirus-britain-vaccine-pa-idUSKBN2B90WN > (last accessed 22 April 2021).

5 New York Times, "Likely legal, 'vaccine passports' emerge as the next coronavirus divide" (New York Times, 6 April 2021) <https://www.nytimes.com/2021/04/06/us/politics/vaccine-passports-coronavirus.html> (last accessed 22 April 2021).

6 Euronews, "WHO advises against vaccine passports as EU debates 'Green Pass"” (euronews.com, 18 March 2021) $<$ https://www.euronews.com/2021/03/18/who-advises-against-vaccine-passports-as-eu-debates-green-pass $>$ (last accessed 22 April 2021).

7 Institute for Government, "Vaccine passports" (The Institute for Government, 24 March 2021) <https://www. instituteforgovernment.org.uk/explainers/vaccine-passports> (last accessed 22 April 2021).

8 Good Health Pass, "Good Health Pass Collaborative - a safe path to global reopening" $(2021)<$ https://www. goodhealthpass.org/> (last accessed 22 April 2021).

9 E Renieris, "The dangers of blockchain-enabled 'immunity passports' for COVID-19" (Medium.com, 18 May $2020)<$ https://medium.com/berkman-klein-center/the-dangers-of-blockchain-enabled-immunity-passports-for-covid19-5ff84cacb290> (last accessed 22 April 2021).

10 WHO, “'Immunity passports' in the context of COVID-19" (who.int, 24 April 2020) < https://www.who.int/newsroom/commentaries/detail/immunity-passports-in-the-context-of-covid-19> (last accessed 22 April 2021).
} 
WHO has clarified that it is in favour of digital vaccination certificates, but not of making these a prerequisite for international travel, ${ }^{11}$ as it might result in "discrimination against the people who are not able to have the vaccine for one reason or another". ${ }^{12}$ The African Union (AU), representing the region with the lowest rates of vaccination and, arguably, the least resilience to the economic and social shocks of the pandemic, is sponsoring a travel certification system for vaccinated people "to promote hassle-free travel across Africa while, at the same time, preventing cross-border spread of COVID-19 infection". ${ }^{13}$ The AU's language about the rationale for certification is particularly surprising given the experience of Ebola, where attempts to formally control West Africa's extremely porous borders were almost entirely ineffective in preventing the spread of the disease. ${ }^{14}$

This article examines vaccination and immunity certificates as the latest entry in a series of risk-reduction technological solutions that, from the start, have characterised the response to the COVID-19 crisis. Nationwide contact tracing apps monitor public health and regulate sociality. Predictive analytics software support policymakers in strategic decisions such as the imposition of lockdowns and curfews. Thermal cameras control access to public spaces such as airports and events. These tools have in common the ability to generate data that result in monitoring or decision-making. They form part of an ever-growing set of data infrastructure with regulatory functions that increasingly constitute the scaffolding of human and social life. In the context of this technologically mediated power over social and economic life, which is now increasingly framed through a health lens, these certification tools are also likely to have disruptive social and political effects.

First, they establish hierarchies between the certified and the uncertified (as has occurred in Israel). Second, they create exclusive markets and power for the actors involved in developing and implementing the technology. Third, and this is perhaps the biggest and least visible risk of all, they appeal to a rhetoric of individual freedom and the "right" to live untrammelled by COVID-19 restrictions: a rhetoric that undermines the solidarity necessary to combat the negative effects of the pandemic on communities local, national or global. If the pandemic becomes something that the wealthy and powerful no longer need to consider, this is problematic not only for rebuilding our damaged economies and societies, but also for sustaining the conditions of cooperation necessary to manage international cooperation, including around the risks of future pandemics. Indeed, as Tazzioli points out in this issue, the effects of the pandemic on bordering practices actively reinforce "class-based and racialised restrictions", while Häkli, also in this issue, argues that this reinforcement of already unjust distinctions expands the category of "irregular migrant" to include anyone whose digital COVID-19 status is unclear (or malfunctioning), making the individual's link to verification infrastructures a subject for the exercise of

\footnotetext{
11 Euronews, supra, note 6.

12 Reuters, supra, note 4.

13 AfricaCDC, "Trusted travel - Africa CDC" (2021) <https://africacdc.org/trusted-travel/> (last accessed 22 April 2021).

14 LD Valdez et al, "Predicting the extinction of Ebola spreading in Liberia due to mitigation strategies" (2015) 5 Scientific Reports 12172.
} 
sovereignty. Below, we analyse how certification systems bring together rhetorical performance with technical performativity to create and justify concrete mechanisms of discrimination, inequality and exclusion.

\section{Discussion}

The process of conceptualising, developing and deploying immunity certification is not a purely technical one. The narratives and negotiations involved in establishing a certification regime involve a strong element of performance that is linked to national and international political orders. Those in charge of developing the intervention publicly perform the rationale for its effectiveness and desirability so as to achieve public buy-in and participation, as well as to build alliances and expand the scope of the project. This performance, aligned with the "security theatre" identified by Schneier in the wake of the $9 / 11$ attacks in the USA, ${ }^{15}$ has various aims. It highlights action and activity and creates the impression of effectiveness on the part of government, while discouraging critique and resistance. In the case of immunity certification specifically, incomplete and constantly developing scientific knowledge regarding the epidemiological processes involved in COVID-19 transmission meant that this technological performance also requires, and feeds on, the suspension of disbelief regarding existential uncertainty on the part of both the public and those devising the intervention. This approach aligns with McGoey's theorisation of strategic ignorance ${ }^{16}$ and Scheel and Ustek-Spilda's ${ }^{17}$ "productive nature of ignorance and nonknowledge", where "ignorance [is] a particular type of nonknowledge that is actively produced as it involves the obfuscation or suppression of otherwise available knowledge". In this case, strategic ignorance of continually updating scientific evidence is often required for people to buy into policy and technical approaches such as certification, because certification performs security in relation to COVID-19 regardless of the efficacy or utility of the actual interventions involved. We argue here that immunity certification constitutes the production of certainty through performance by using an engineering definition of immunity to achieve the political acceptance of new systems of inclusion and exclusion. By doing so, it also actively distracts both attention and resources from the real goals of protecting people from infection and mitigating the spread of the COVID-19 virus.

Beyond performance, we can also identify immunity certification as involving performativity - a notion originally defined by Butler ${ }^{18}$ as the reification of gender through behaviour, expression and performance, and since developed in many different domains of theory. The establishing of immunity certification systems goes, nevertheless, beyond performance in order to gather support for these systems as ways of organising and governing behaviour and mobility. Instead (or as well), it

15 B Schneier, Beyond Fear: Thinking Sensibly about Security in an Uncertain World (Berlin, Springer Science+Business Media 2006).

16 L McGoey, "Strategic unknowns: towards a sociology of ignorance" (2012) 41 Economy and Society 1.

17 S Scheel and F Ustek-Spilda, "The politics of expertise and ignorance in the field of migration management" (2019) 37 Environment and Planning D: Society and Space 663.

18 J Butler, Gender Trouble: Feminism and the Subversion of Identity (London, Routledge 2011). 
concretely establishes immunity to COVID-19 as a possibility, stabilising what is a discursive object of scientific research into an attribute that carries across contexts and applications. In comparison to past formalisations of the notion of immunity, such as vaccination for the flu or for childhood diseases, COVID-19 certification creates a new kind of immunity: one that focuses not on the scope of vaccination as a source of protection, but on the notion - formerly a probabilistic one that did not involve a claim to certainty - that a particular individual is innocuous and will remain so. This stamp effectively introduces the idea for the first time that someone can be certified as immune, rather than just vaccinated. This constitutes a new, commoditised identity that, in turn, has value for both the individual and the authorities certifying them - a value that is different from the more general, public health-related contribution of decreasing the communicability of COVID-19 overall.

We can also identify the ways in which, in the case of certification, the systems and infrastructures involved and the claims that attach to their use do not merely perform the aspirations of policy but change the world in material ways, creating different possible futures. Certification systems, as currently being proposed and deployed, actively stabilise what is uncertain and provide fixed points of perceived truth and infrastructural capacity from which policy can operate. Less visibly, they will also shape the codespace we inhabit ${ }^{19}$ in far-reaching ways, interacting with the performance and rhetoric that do the work of establishing and sustaining support for this concrete power.

In what follows below, we analyse technology as performative, as well as performance, looking at both what Mackenzie ${ }^{20}$ has termed "the performative constitution of collective agency" around certification projects, and what Amicelle et $\mathrm{al}^{21}$ describe as the performative power of technological devices, which "redraw the boundaries between the normal and the abnormal, introducing substantive forms of inequality in the process". We argue that this process of building new and undesirable futures using immunity certification infrastructures does not have to be strategic in order to be problematic or worth resisting: to do so, we will focus in on various aspects of these forms of performance and performativity, exploring how they add up to new futures that should, in many cases, be resisted.

\section{Verification theatre}

Verifying identity and attesting to possessing certain attributes happen in many contexts. While the stakes depend on individual circumstance, taking out a library book will generally call for a lower standard of proof than emptying a bank account. Approaches to verification are connected to the level of proof needed. Society is willing to accept a theoretically higher risk of stolen library books than emptied bank accounts (false accepts) and willing to accept more false rejects (where individuals

\footnotetext{
19 R Kitchin and M Dodge, Code/Space: Software and Everyday Life (Cambridge, MA, MIT Press 2014).

20 A Mackenzie, "The performativity of code: software and cultures of circulation" (2005) 22 Theory, Culture \& Society 71 .

21 A Amicelle, C Aradau and J Jeandesboz, "Questioning security devices: performativity, resistance, politics” (2015) 46 Security Dialogue 293.
} 
entitled to access are incorrectly barred out of an abundance of caution) in banking than in library usage. Yet false rejects do not merely have impacts on convenience, but can also shape universal access and discrimination, as the cost and likelihood of rejection is unlikely to fall equally or randomly, with the cumulative effects of such inequalities leading to significant injustice. ${ }^{22}$

Where does immunity certification fall on such a spectrum, and where should it fall? Vendors of immunity certification systems (often already providers of identity) suggest that a (if not the) core issue is the question of fraud, which they claim a technical solution would prevent. ${ }^{23}$ However, on closer inspection, this claim and framing represent an elaborate performance with unreal properties.

Neither vaccinations nor COVID-19 testing have been routinely delivered in a context of robust identity authentication. Consequently, there is a familiar problem - garbage in, garbage out. Both have been administered in hastily constructed venues and home delivery or visit services (rightly) designed primarily for speed, capacity and safety. As such, most such interventions were not primarily preoccupied with verifying that the people receiving the treatment were who they said or thought they were. Tens or hundreds of thousands of individuals will be in difficult-to-prove or unconventional situations, such as being part of clinical vaccination trials or having received a test or one or more vaccine shots abroad before moving. Such variable attributes would need to be factored in if a system is to properly "account" for the populations using it - a necessarily lax procedure due to the large number of edge cases. What is more, the number of individuals authorised to verify a vaccine or test related to a particular person will easily number in the hundreds of thousands, with rapid turnover, particularly once the need for international interoperability for travel and tourism, a prime motivator, is considered. Additionally, vaccines and tests that have already been administered risk being "too late" to add identity authentication to them, and attempts to increase the security of further processes seem ethically questionable due to both deterrence effects on communities concerned about state surveillance or lacking authentication, as well as the general public health implications of slowing down a public health intervention in order to attempt to prevent downstream fraud. Regardless of how difficult a vendor makes it to slip through digital attribute authentication when an individual is holding an app, the attributes that app draws upon cannot themselves be trusted.

Secondly, anticipated use cases for these certificates have, in many sectors, limited to no capacity or incentives to robustly use even attribute authentication systems that do exist. The EU's hospitality sector, for example, having already haemorrhaged resources for over a year, is hardly in a position to start employing special concierges for this task, particularly as COVID-19 adjustments, such as table service or cleaning,

22 OH Gandy, Coming to Terms with Chance: Engaging Rational Discrimination and Cumulative Disadvantage (London, Routledge 2016).

23 S Venkataramakrishnan and A Gross, "COVID-19 vaccine passports 'should not last for ever', says UK developer" (ft.com, 3 April 2021) <https://www.ft.com/content/8d6479f3-62e8-4232-91a8-c05ed4acbe90> (last accessed 22 April 2021); D Diamond, “'Ripe for fraud': coronavirus vaccination cards support burgeoning scams" (Washington Post, 18 April 2021) < https://www.washingtonpost.com/health/2021/04/18/scams-coronavirus-vaccination-cards/> (last accessed 22 April 2021). 
are already incurring significant additional costs. Given this sector's economic duress, it is also unlikely to refuse customers without a significant national (or European) punitive regime in place, sufficiently policed. Even when the will to ascertain COVID-19 safety exists, hospitality and leisure venues are not architecturally designed for security. Determined individuals need only slip in a side route or exit. Some exceptions exist: already-securitised places such as airports or large event venues may be able to roll certificates into border- or ticket-checking procedures. Yet the popular idea that a much larger collection of services become conditional on immunity attestation does not appear either physically or economically realistic.

While the current vendors of identification infrastructure act as if a technical system can be made that in the short to medium term can prevent individuals with mild or high determination from, for example, successfully entering a restricted location, this is an elaborate performance. Technology may be able to add hurdles for those who wish to bypass a given rule, but these hurdles do not appear particularly daunting. In situations such as this, legal provisions, which make fraud costlier due to risk of a prosecution, are typically fallen back upon. However, the motivation to promote elaborate technical approaches, regardless of how they might in practice exclude individuals or populations, is high. Identity vendors have a vested interest in identifying COVID-19 as a context that is high risk, requiring few false accepts. In order to achieve this, a necessary but insufficient criterion is secure attribute authentication - the association between a physical individual and a recorded attribute (eg relating to reduced transmission of COVID-19). This typically has two stages: identity authentication (the person in front of me is associated with a given identifier) and authenticating an attribution-identifier association (a given identifier is associated with a recorded attribute). This can happen facilitated by one tool (eg a passport biometrically facilitates identity authentication as well as proving citizenship) or by combining documents (eg a WHO International Certificate of Vaccination or Prophylaxis is presented to show a vaccine associated with the identifier of a name, while another document, such as a passport, is used to associate the individual with that name).

Digital identity vendors only selling attribute-identifier association are, in fact, easily supplanted by anyone with an authoritative database. The real prize is, therefore, to control identity authentication. Attribute-identifier databases have to utilise those vendors' identifiers, placing them in the position of a difficult-to-displace intermediary (or even a critical infrastructure) - a position from which economic value can be extracted from a variety of sources, in myriad ways. In this sense, the performance of digital security in the COVID-19 pandemic, while having little added benefit (and potentially bringing exclusion and other serious concerns), does something very "real" and significant in the world. It risks enacting an open-ended, digital and largely privatised infrastructure for proving things to entities. If certification systems are to be built and deployed at speed, the central role of the private sector in much of the Global South, and especially smaller countries, is a near certainty due to limited state capacity or funding to develop technology in-house in a rapid way that interoperates with other parts of the world. 


\section{Immunity theatre}

The concept of immunity has emerged as deeply theatrical during this pandemic. What exactly leads individuals to be less able to transmit the disease is both uncertain and a moving target. Immunity certificate proposals to date include: vaccinations; booster doses; current polymerase chain reaction (PCR) or lateral flow tests; proof of previous diagnoses; or antibody tests. Each of these may have further relevant data attached, relating to variants or batches of viruses, tests or interventions and the moment in which each occurred. Transmissibility might even be analysed as a function of one or more of these attributes and treated as a quantitative risk profile. Despite a preponderance of indicators, there are both serious limits to what we know about immunity today and what can be known in relation to the concept tomorrow. Immune response to COVID-19 is difficult to study even in highly controlled environments, although new "Challenge Trials" seek to purposefully expose individuals to the virus in order to create cleaner experimental conditions. The most recent research at the time of writing ${ }^{24}$ finds that the ability to sustain immunity to COVID-19 for up to ten months is highly dependent on (older) age, disease severity, gender (with men sustaining immunity better than women) and the symptoms experienced during infection. This indicates both that there is high variability in people's immune responses and that these answers will continue to be shaped by new information. Furthermore, studies conducted on vaccinated populations show that those most vulnerable to COVID-19 remain substantially vulnerable after vaccination. ${ }^{25}$ Moreover, immunity status is realistically less relevant than transmissibility status, which, as the public are now well aware, is not always tied to exhibiting symptoms.

It is important to also note here that transmissibility is not a status that relates to an individual, but an individual in context. The Netherlands and the UK are among a number of countries running large "test events" that claim to attempt to quantify transmission in group situations. However, given that everyone attending such "experimental" events is PCR-tested for COVID-19 immediately beforehand, the events only demonstrate that PCR tests are able to detect active infection, and that as long as those testing positive are reliably excluded, gatherings will not result in new infections. The "experimental" theatre of having some participants wear masks or socially distance while others do not does not appear to have any medical research value, but can instead be described as a political and business experiment: it demonstrates that event organisers can do business as long as they only open their doors to people who have definitely been tested for COVID-19 in the last twenty-four hours. ${ }^{26}$

\footnotetext{
$24 \mathrm{~K}$ Vanshylla et al, "Kinetics and correlates of the neutralizing antibody response to SARS-CoV-2 infection in humans" Cell Host \& Microbe doi: 10.1101/2021.01.26.428207.

25 Medicalxpress.com, "Study probes COVID among vaccinated at US nursing home" (Medicalxpress.com, 22 April 2021) < https://medicalxpress.com/news/2021-04-probes-covid-vaccinated-nursing-home.html> (last accessed 14 May 2021).

26 In the Dutch context, the "Fieldlab" experiments have come under significant critique as lacking any scientific basis: NRC, "Fieldlabfeest 538 Oranjedag Krijgt Geen Vergunning van Gemeente" (NRC, 19 April 2021) <https:// www.nrc.nl/nieuws/2021/04/19/coronablog19-a4040317\#upd20210419171711> (last accessed 22 April 2021).
} 
Finally, immunity is a moving target, as immune responses fade and variants mutate and escape existing protection. All of these factors mean that immunity for the purpose of certification in society is not a static status that relates to an individual, but at best an uncertain, contextually situated cluster of indicators primarily relating to a best guess at transmission risk.

\section{Border theatre}

Certification can also be seen as a form of bordering activity, which, as Amoore and Hall $^{27}$ have shown, always includes theatrical aspects. Bordering, ${ }^{28}$ or the set of activities involved in constructing and managing international borders, takes place both at national borders and within countries where migration is governed through restrictions and rules for particular populations. The management of borders can be seen as a form of theatre: today's borders are performed through the deployment of biometric technologies in passport and customs checks, through spatial monitoring by cameras, satellites and other sensing devices and through many other technological devices. As also argued by Häkli in his contribution to this special issue, immunity certification technologies promise to become a form of bordering, linking up public health monitoring technologies with the existing border apparatus. Like that existing apparatus, however, certification systems will be both probabilistic and porous people will find their way across borders by faking documentation, through technical faults and through human error. The theatre involved in bordering - visible technologies of identification, verification and analysis - has perhaps its most important function in helping authorities seem more effective, and by doing so dissuading some of those who might try their luck at gaming the system in order to transit irregularly. Certification goes beyond this in adding a new and less negotiable layer to the permissions and prohibitions involved in migrating across borders and within countries as a foreigner: it creates a new logic for stopping and searching people on the basis of possible origin, something that domestic law has made less easy, but that can be easily reinstated with emergency public health measures.

\section{Equality theatre}

Along with the developments outlined above, it is possible that the most far-reaching effects of immunity certification may be on global inequality, where they promise to enact both logics and practices of inclusion and exclusion based on probabilistic and shifting distinctions. The international community is keen to deflect accusations of reproducing and increasing inequality, as can be seen from the underfunded and (at the time of writing) underwhelming ${ }^{29}$ COVAX programme of vaccine sharing between higher- and lower-income countries. This rhetorical deflection of

27 L Amoore and A Hall, "Border theatre: on the arts of security and resistance" (2010) 17 Cultural Geographies 299.

28 D Newman, "Borders and bordering: towards an interdisciplinary dialogue" (2006) 9 European Journal of Social Theory 171.

29 The Guardian, "Revealed: big shortfall in Covax Covid vaccine-sharing scheme" (The Guardian, 22 April 2021) $<$ https://www.theguardian.com/world/2021/apr/22/revealed-big-shortfall-in-covax-covid-vaccine-sharing-scheme> (last accessed 22 April 2021). 
responsibility for amplifying deeply embedded historical and structural inequalities between nations and populations is perhaps most visible in the equality theatre of the WHO's published requirements for the technological underpinnings of an international certification system. ${ }^{30}$ None of the risks of increasing inequality, including those pointed out in 2020 by the WHO itself, had been addressed at the point where applications to join the international consortium were opened. Nevertheless, the tendering process required applicants to confirm that they would design systems that would not create inequality in access to certificates (despite the clear global inequality of access to vaccines), or in fact inequities in general, specifying as requirements that "Everyone has the right to obtain and hold a smart vaccination certificate" and that "The smart vaccination certificate should not be increasing health inequities or increasing the digital divide". 31

The equality theatre that frames the designers of technical systems as those who can solve inequality is an undesirable form of theatre not only because those actors are not the ones relevant to solving it, but also because they are in fact those most likely to be the ones actively exacerbating it. More importantly, this theatre implies both that certification systems can optimise for equality as long as they are designed correctly and that this is an appropriate way to address what, in the case of COVID-19, has amounted to structural violence perpetrated against the world's most disadvantaged and marginalised people. This kind of theatre is not new: it is common to innumerable "techno-saviourist" 32 interventions where a new technology is posited as solving a complex, historically rooted and structural problem in a way that distracts from actually addressing it. Examples of this include claiming that a particular brand of cooking stove will reduce gender inequality and, specifically, the prevalence of rape in post-conflict settings, ${ }^{33}$ or the "frugal thermometer" that developers promise will counter the problem of a lack of healthcare resources in low-income countries. ${ }^{34}$

\section{Conclusion}

This article has explored the ways in which proposed and deployed systems for immunity certification both constitute forms of theatre designed to open up particular courses of action and behaviour and to stabilise scientific uncertainty into manageable policy actions and constitute real-world alliances and infrastructures that make possible particular futures and close off others. Their impact on the spread and control of

\footnotetext{
30 WHO, "World Health Organization open call for nomination of experts to contribute to the smart vaccination certificate technical specifications and standards" (who.int, 12 February 2020) <https://www.who.int/news-room/ articles-detail/world-health-organization-open-call-for-nomination-of-experts-to-contribute-to-the-smart-vaccinationcertificate-technical-specifications-and-standards-application-deadline-14-december-2020> (last accessed 22 April 2021).

31 ibid.

32 S Abdelnour, "The cookstove-rape prevention myth and the limits of techno-saviorism" in S Hostettler, A Gadgil and E Hazboun (eds), Sustainable Access to Energy in the Global South (Berlin, Springer 2015) pp 205-15.

33 S Abdelnour and AM Saeed, "Technologizing humanitarian space: Darfur advocacy and the rape-stove panacea" (2014) 8 International Political Sociology 145.

34 CFIA, "Frugal thermometer" (Centre for Frugal Innovation in Africa, 2021) < https://www.cfia.nl/projects/frugalthermometer> (last accessed 4 May 2021).
} 
COVID-19 is likely to be negligible given that they rely on notions of immunity that are at best scientifically unsupported and at worst designed to facilitate policy rather than control disease. This driving function of performing and embodying policy indicates, indeed, that these systems should be addressed as instruments not only of public health, but also of migration policy, technology and economic policy and law enforcement policy, to name but the most obvious. It is because of these functions that we should care about the disparate impacts that immunity certification will have at the global level. We should expect them to be enacted at different scales, some of which (such as workplaces, restaurants and sports events) will be under the control of legal authorities tasked with protecting rights and guarding the public interest, and some of which (such as international migration policy, global public health measures and international technology markets) will be very far from the control of any authority guided by human rights or concerns of equity. In the international arena, it is geopolitical power that counts, and immunity certification risks furthering the interests of the most powerful at the expense of other nations. Those whose ability to travel to, study in and unite with family members in high-income countries is already highly constrained may see it reduced to zero due to the unequal distribution of vaccines (or the right type of vaccine) and accredited testing facilities or simply the devices and connectivity to use certification systems. It is not only the individual at the end of the chain who needs the right devices and connectivity infrastructures and services, but everyone involved in testing, vaccinating and attesting that an individual is eligible to be certified. In large swathes of the world, these devices and connectivity are lacking. In the context of this kind of inequality, standardisation is neither possible nor equitable because it would inevitably play to the interests of those with the greatest clout in international affairs.

The proposed (and deployed) COVID-19 immunity certification programmes depart from the established logic of immunity certification (eg with the common yellow fever certificate or childhood vaccination proof), where the aim is to create solidarity around the common good of vaccination and herd immunity. With COVID-19 certification, the aim is to privilege some individuals over other individuals, which goes against every former aim of such programmes. For this reason, regardless of its probably technical dysfunctionality, the platformisation of attribute authentication cannot lead to equity between countries, populations or groups. Instead, it will help to stabilise a global caste system where certain groups are unable to travel legally, work legally or gather legally, and in doing so it will distract attention and activity away from redressing this inequality. 